BMJ

Open

Gastroenterology

\section{Novel multidisciplinary hub-and-spoke tertiary service for the management of severe acute pancreatitis}

To cite: Nayar MK, Bekkali NLH, Bourne D, et al. Novel multidisciplinary hub-andspoke tertiary service for the management of severe acute pancreatitis. BMJ Open Gastro 2021;8:e000501. doi:10.1136/ bmjgast-2020-000501

\section{- Prepublication history and additional supplemental material is published online only. To view please visit the journal online (http://dx.doi. org/10.1136/bmjgast-2020- 000501).}

Received 27 July 2020 Revised 26 November 2020 Accepted 28 February 2021

\section{Check for updates}

(C) Author(s) (or their employer(s)) 2021. Re-use permitted under CC BY-NC. No commercial re-use. See rights and permissions. Published by BMJ.

For numbered affiliations see end of article.

Correspondence to Dr Manu K Nayar; manu.nayar@nhs.net

\section{ABSTRACT}

Objective Severe acute pancreatitis (SAP) is associated with high mortality (15\%-30\%). Current guidelines recommend these patients are best managed in a multidisciplinary team setting. This study reports experience in the management of SAP within the UK's first reported hub-and-spoke pancreatitis network.

Design All patients with SAP referred to the remote care pancreatitis network between 2015 and 2017 were prospectively entered onto a database by a dedicated pancreatitis specialist nurse. Baseline characteristics, aetiology, intensive care unit (ICU) stay, interventions, complications, mortality and follow-up were analysed. Results 285 patients admitted with SAP to secondary care hospitals during the study period were discussed with the dedicated pancreatitis specialist nurse and referred to the regional service. $83 / 285$ patients (29\%; 37 male) were transferred to the specialist centre mainly for drainage of infected pancreatic fluid collections (PFC) in $95 \%(n=79)$ of patients. Among the patients transferred; $29(35 \%)$ patients developed multiorgan failure with an inpatient mortality of $14 \%(n=12 / 83)$. The median followup was 18.2 months (IQR=11.25-35.51). Multivariate analysis showed that transferred patients had statistically significant longer overall hospital stay $(p<0.001)$ but less ICU stay $(p<0.012)$.

Conclusion This hub-and-spoke model facilitates the management of the majority of patients with SAP in secondary care setting. $29 \%$ warranted transfer to our tertiary centre, predominantly for endoscopic drainage of PFCs. An evidence-based approach with a low threshold for transfer to tertiary care centre can result in lower mortality for SAP and fewer days in ICU.

\section{INTRODUCTION}

Acute pancreatitis is a common presentation and most hospitals in the UK serving a population of 300000-400000 admit around 100 patients on a yearly basis. ${ }^{1}$ Severe acute pancreatitis (SAP) which occurs in $20 \%$ of patients is characterised by persistent organ failure and often requires critical care support, endoscopic or radiological intervention in $40 \%$ of patients. ${ }^{2-6}$ Endoscopic

\section{Summary box}

What is already known about this subject?

- Management of severe acute pancreatitis (SAP) requires a multidisciplinary approach with access to specialist regional services including intensive care facilities and expertise in endoscopic management of pancreatic necrosis.

- In spite of recommendations from multiple international guidelines (European Society of Gastrointestinal Endoscopy, National Institute for Health and Care Excellence and National Confidential Enquiry into Patient Outcome and Death report), it is challenging to set up such a regional service for optimum care for the patient. In addition, there are limited data in literature around this topic.

What are the new findings?

- This is the first reported hub-and-spoke network in the UK for the management of SAP.

- The new findings of the service include a coordinated multispecialty approach from referral to discharge by a dedicated pancreatitis specialist nurse, regular feedback to referring units on a weekly basis and transfer of patients in a timely manner if intervention is required.

- An evidence-based approach enables regular contact with the referring unit ensuring a high standard of care, avoids unnecessary transfer of patients and keeps the patient closer to home.

How might it impact on clinical practice in the foreseeable future

- This study concludes that a dedicated multidisciplinary hub-and-spoke pancreatitis network results in optimal and a high standard of care of patients with SAP.

This will enable pancreatic units to establish similar hub-and-spoke patient care pathways for management of this sick group of patients.

or radiological intervention is recommended in patients with proven or suspected infected necrosis, bleeding or abdominal compartment syndrome ${ }^{7-9}$ However, despite 
treatment, the mortality of severe necrotising pancreatitis is $15 \%-30 \%$. In the UK, the National Confidential Enquiry into Patient Outcome and Death (NCEPOD) report from 2016 recommended a multidisciplinary team (MDT) approach in acute pancreatitis following a review on quality of care of UK patients with acute pancreatitis. ${ }^{17}$ In addition, the recent National Institute for Health and Care Excellence (NICE) guideline from 2018 recommended regional networks and/or 'hub-andspoke models' for these patients to ensure adequate and timely interventions. ${ }^{23}$ One of the challenges of setting up such a service is the lack of a single point of contact coordinating the management of this complex group of patients and a need to keep them in a healthcare setting close to home for as long as possible.

In 2015, we set up the first regional pancreatitis service in the North East of England. The service provides a huband-spoke model for the management of SAP. Patients warranting endoscopic, surgical or radiological intervention are transferred to the tertiary referral centre for further management. We report our experience of the management of patients with SAP within the regional hub-and-spoke pancreatitis network.

\section{METHODS}

\section{Patients and pathway}

The North East regional of the England comprises a population of around 3.5 million people over a large geographical area. Within this area, there are 17 secondary care hospitals which admit patients with acute pancreatitis. The Freeman Hospital in Newcastle upon Tyne is the only dedicated pancreatic centre for this region providing a full range of intensive care unit (ICU) facilities and interventions including endoscopic, surgical and radiological intervention. An ad hoc service was first established in 2013 and regional protocols were developed for patients with SAP based on the best available current evidence including ICU management, timing of intervention, use of antibiotics and nutritional supplementation. This was developed as a remote care pancreatitis (RCP) service, that is, patients admitted with SAP were referred to our service and we managed the patients remotely with weekly discussions and feedback to the secondary care team looking after the patient till it was deemed necessary to transfer the patient to our unit for specialist management. The service was subsequently formalised in 2015 with weekly discussions in a team consisting of a dedicated pancreatitis nurse specialist (SY), pancreatic radiologists (JS), hepatopancreatobiliary (HPB) surgeons (RMC, JJF, GS, JLL and SP), HPB physicians (KWO, MKN and JSL) and a pancreatic dietician (DB).

Data on patients aged $>18$ years with SAP (as defined by the Atlanta classification $2012^{10}$ who were referred and admitted to Freeman hospital between June 2015 and July 2017) were collected from a prospectively maintained database. The severity of pancreatitis is based on the revised Atlanta criteria. Patients with severe pancreatitis with necrosis are referred to the RCP service for further input. The referring centres are aware of this indication. Data for the total number of patients admitted with acute pancreatitis during this period were obtained from the regional Hospital Episode Statistic database based on specific code for acute pancreatitis (ICD K85). All patients were discussed in the weekly RCP MDT meeting and only patients requiring endoscopic, radiological or surgical intervention or specialist ICU care were transferred. Baseline characteristics, aetiology, nutritional support, antibiotic treatment, ICU stay, interventions, complications and mortality were recorded. In addition, the reason for transfer as well as time to transfer, location of transfer (to ICU or ward) and length of stay-ICU stay and ward stay - were reported. All patients were followed up for a minimum of 1 year following discharge from hospital.

Patients who were deemed not for transfer, that is, well enough for generic local input and/or not requiring immediate interventions were rediscussed in the weekly MDT . Patients who needed to be transferred were assessed for suitability for ward or ICU transfer. The intensive care team were additionally consulted for those who needed direct ICU transfer. Patients requiring only ICU care locally were not transferred unless intervention was deemed appropriate or if a significant change in ICU management was required. The reasons for such a threshold were twofold: (1) conventional ICUcare without intervention could usually be managed by non-tertiary ICU as per established national guidelines and (2) Given the significant geography of the region covered by the RCP MDM, there remains a preference for family/relatives to keep the patients in a healthcare setting nearer to their home if possible.

The indications for transfer to the Freeman hospital for specialist management included:

1. Critical emergency-haemorrhage or perforation.

2. Deteriorating organ function.

3. Localised collection/walled off necrosis requiring urgent endoscopic drainage.

The pathway for remote care management is shown in figure 1 .

\section{Interventions}

If intervention was deemed necessary following the MDM discussion, percutaneous or endoscopic ultrasoundguided drainage (which is exclusively performed in our centre) or other interventions, that is, radiological embolisation for bleeding or bypass surgery for gastric or biliary obstruction, were performed. Percutaneous drainage as an interim measure was advised to the referring hospitals (where available) if deemed necessary by the MDM.

\section{Statistical analysis}

Patient data were analysed using MedCalc software, V.11.2.1.0 (MedCalc Software, Ostend, Belgium). The data were descriptive and where applicable (eg, age) 


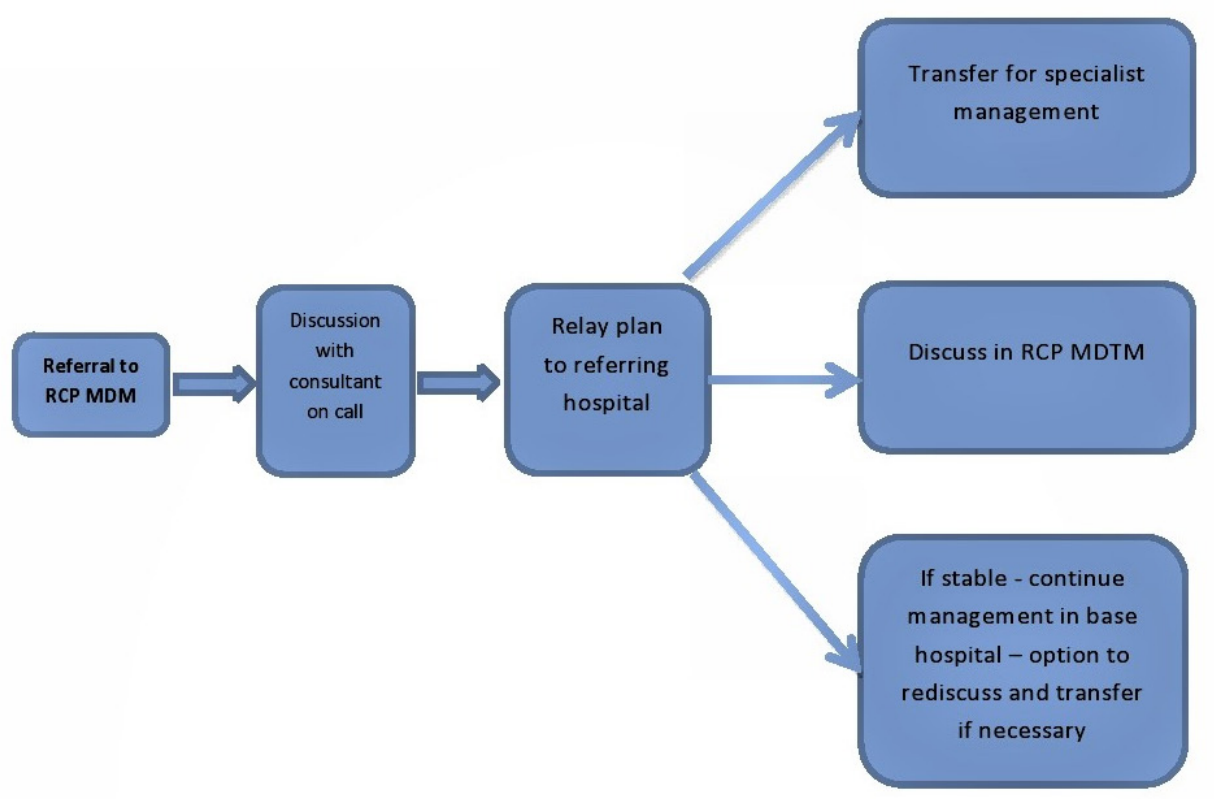

Figure 1 Remote care pathway. RCP MDM, remote care pancreatitis multidisciplinary meeting.

continuous data were provided using the median with IQR. Multivariate analysis was undertaken to compare data between the transferred and the non-transferred patients.

\section{Consent}

Written informed consent was obtained prior to any interventions.

In 2015, the first North East of England RCP service with a dedicated MDT was set up serving a population of 3.5 million and 17 referring hospitals in the region $\left(\right.$ area $\left.=8592 \mathrm{~km}^{2}\right)$.

The pancreatitis specialist nurse has a key role in coordinating the service from the time of referral to the MDT and subsequent transfer of patients to our tertiary service. The initial referral is an electronic referral to the pancreatitis specialist nurse who collects all data on baseline characteristics, nutrition, cross-sectional imaging, previous interventions, date of admission and the type of admission (ICU or ward) (online supplemental file 1). Once the mandatory data are collected, the MDM discussion is awaited. If the patient is too unwell to await discussion, the specialist nurse liaises with the on-call HPB-surgeon/physicians and provides telephone feedback to the local hospital with a management plan. Access to the specialist nurse was only available during normal working hours, out of hours, the patient was discussed with the on call HPB team.

The outcome of the weekly MDM feedback is dictated by letter and sent via email to the referring clinicians on the same day. All outcomes contain direct phone numbers to the pancreatic specialist nurse who liaises with the referring team.

\section{RESULTS}

A total of 4683 patients with acute pancreatitis including mild, moderately severe and severe were admitted to the North East of England between June 2015 and July 2017. Of 4683, $285(6.1 \%)$ patients with SAP were referred for discussion to the service for specialist input. Of 285,83 (29\%) patients were transferred to our centre for further specialist management. Demographic data of the two groups of patients are summarised in table 1 .

\section{Outcome of patients transferred to the tertiary specialist centre}

Patients were referred for MDM input to the specialist centre at a median of $8(\mathrm{IQR}=3-16)$ days postadmission locally. The median number of discussions in the MDM meeting prior to transfer was $1(\mathrm{IQR}=1-2)$. The most common reason for transfer from local hospitals to the tertiary care was for drainage of peripancreatic collections in $79(95 \%)$ patients. The remaining four patients were transferred due to the following reasons: specialist management due to complex past medical history, pancreatic ascites, expert ICU supportive care and pancreaticopleural fistula management.

The median time to facilitate transfer to Freeman Hospital after MDM discussion was 3.1 ( $\mathrm{IQR}=1-5)$ days. Of the transferred patients, 26\% needed direct ICU transfer and $35 \%$ patients had multiorgan failure (MOF). All patients with suspected thromboembolic events were anticoagulated.

\section{Nutrition}

Seventy-five $(84 \%)$ patients were orally or enterally fed via nasogastric or nasojejunal tubes, $12(15 \%)$ received parenteral nutrition on transfer to our centre. One 


\begin{tabular}{|c|c|c|c|}
\hline & $\begin{array}{l}\text { Transferred } \\
\text { patients }-83\end{array}$ & $\begin{array}{l}\text { Non } \\
\text { transferred } \\
\text { patients }-202\end{array}$ & $\begin{array}{l}P \\
\text { value }\end{array}$ \\
\hline \multicolumn{4}{|l|}{ Sex } \\
\hline Male & 37 & 114 & 0.89 \\
\hline Female & 46 & 88 & \\
\hline Median age (IQR) & $\begin{array}{l}56.5(40.0- \\
70.0)\end{array}$ & $\begin{array}{l}57.5(47.0- \\
69.0)\end{array}$ & 0.65 \\
\hline Aetiology & 26 & 70 & 0.458 \\
\hline Gallstones & 37 & 69 & \\
\hline Alcohol & 14 & 46 & \\
\hline Idiopathic & 4 & 8 & \\
\hline Post-ERCP & 0 & 4 & \\
\hline Drug induced & 0 & 2 & \\
\hline \multicolumn{4}{|l|}{ Hyperlipidaemia } \\
\hline $\begin{array}{l}\text { Acute-on-chronic } \\
\text { pancreatitis }\end{array}$ & 1 & 2 & \\
\hline Post-EUS & 0 & 1 & \\
\hline Postoperative & 1 & 0 & \\
\hline $\begin{array}{l}\text { Median days } \\
\text { admission to } \\
\text { referral }\end{array}$ & $8.0(3.0-16.0)$ & $7.0(4.0-11.0)$ & 0.25 \\
\hline $\begin{array}{l}\text { Median no of times } \\
\text { discussed }\end{array}$ & $1.0(1.0-2.0)$ & $1.0(1.0-2.0)$ & 0.1 \\
\hline
\end{tabular}

ERCP, endoscopic retrograde cholangiopancreatography; EUS,

Endoscopic ultrasound; IQR, inter quartile range; no, number.

patient $(1 \%)$ had inadequate indication for parenteral nutrition which was stopped and the patient was started on oral nutrition.

\section{Antibiotics}

Fifty-five patients $(66 \%)$ were on antibiotics of whom $31 / 55(56 \%)$ had positive cultures at the time of transfer -11 blood cultures and miscellaneous $=20$ (sputum, urine, line-tip, wound)). Therefore, 24 (44\%) of patients had inappropriate use of antibiotics which were stopped at transfer.

\section{Interventions}

A total of $18 \%(15 / 83)$ patients had interventions locally before transfer to our centre. The local intervention comprised percutaneous drains in $11(73 \%)$ patients, vascular treatment (embolisation) in $2(13 \%)$ patients, pleural effusion drainage in $1(7 \%)$ patient and percutaneous necrosectomy in one patient $(7 \%)$.

Following transfer, $14(17 \%)$ had spontaneous resolution of pancreatic fluid collections (PFC) following a period of watchful wait and supportive care. Sixty-seven (79\%) patients required drainage as shown in table 2. In addition, vascular embolisation was required in 10 $(12 \%)$ patients with gastroduodenal or splenic artery pseudoaneurysms.
Table 2 Intervention on patients transferred

\begin{tabular}{ll} 
Type of interventions & $\begin{array}{l}\text { Patients } \\
(\mathbf{n}=67),(\%)\end{array}$ \\
\hline Endoscopic drainage (E), including ERCP(3) & $31(46)$ \\
\hline Percutaneous (P) & $19(28)$ \\
Surgical (S) & $3(5)$ \\
Endoscopic+percutaneous & $6(9)$ \\
Endoscopic+surgical & $2(3)$ \\
\hline Percutaneous+surgical & $3(4)$ \\
\hline E+P+S & $2(3)$ \\
Pancreatic ascites drainage & $1(2)$ \\
\hline
\end{tabular}

ERCP, endoscopic retrograde cholangiopancreatography.

\section{Complications}

Overall $30(36 \%)$ patients developed complications as a result of SAP. Of 30, $20(66.7 \%)$ developed complications as a result of intervention including $10 \mathrm{in}$ patients treated endoscopically, 8 percutaneously and 2 due to surgical interventions. The remaining complications (33.3\%) were as a result of the severity of disease which included four pseudoaneurysms, one Clostridium difficile infection, three sepsis, one haemorrhagic pancreatic collection and one veno-occlusive disease.

In the $10(33 \%)$, treated endoscopically, the complications were: sepsis in four patients, two pseudo aneurysms necessitating vascular embolisation, two veno-occlusive disease, one ischaemic colitis treated conservatively and one had sepsis as well as a pseudoaneurysm. Complications in the eight patients treated via the percutaneous route were: two pseudoaneursyms necessitating vascular embolisation, three veno-occlusive diseases, one sepsis, one gastric outlet obstruction and one had cholecystitis treated with antibiotics. The two patients treated surgically had enterocutaneous fistula and one had a pseudoaneurysm necessitating vascular embolisation.

\section{Length of stay}

The median length of stay in the ward and ICU were 61 days $(\mathrm{IQR}=37-97)]$ and $12(\mathrm{IQR}=4-21)$ days, respectively. When excluding patients who died, the median length of stay in the ward and ICU was $51(\mathrm{IQR}=31-82)$ days and 6 $(\mathrm{IQR}=0-18)$ days, respectively.

\section{Mortality}

Twelve patients (14\%) died during their hospital stay. The cause of death in 10 patients was MOF and in 2 patients pulmonary embolism. Of 12,11 (92\%) deaths were in ICU.

\section{Follow-up}

The mean follow-up time for patients transferred to Freeman hospital was 19.39 months (IQR 11.57-27.95). Elective cholecystectomy was performed in 20 of 37 $(54 \%)$ with biliary pancreatitis; 17 were deemed unfit for surgery. New onset of diabetes mellitus was diagnosed in $9(11 \%)$ patients and $71(86 \%)$ had treatment for 
pancreatic exocrine insufficiency with pancreatic enzyme supplements during follow-up. Six patients $(7 \%)$ developed recurrent pancreatitis during the follow-up.

\section{Outcome of patients who were not transferred}

The demographics of patients who were not transferred are shown in table 1 . The median number of times the case was discussed at the MDM was 1 (IQR=0-2). The median length of stay at the referring hospital was 19 days $(\mathrm{IQR}=17-28)$ of which $39 \%(77 / 202)$ were admitted in ICU. Median length of stay in ITU was 8 days $(\mathrm{IQR}=4-17)$.

Data on antibiotic use were available on 199/202 patients. Forty-one per cent were on antibiotics but only $11 \%$ had positive cultures.

Data on nutrition requirements were available for 192/202 patients. Eighty-four per cent of patients were either on oral or enteral tube feeding. Parenteral nutrition during admission was given to $16 \%$ patients. Data on inappropriate use of $\mathrm{PN}$ were not available.

The median follow-up was 18.2 months ( $\mathrm{IQR}=8.2-$ 25.32). Of 202, 34 (18\%) died during the follow-up due to unrelated causes. In-hospital mortality was $8.4 \%$. The most common cause (94\%) was MOF. Overall mortality was $10 \%(29 / 285)$.

On multivariate analysis comparing the two groups, there was no difference age/gender distribution, aetiology and median days from admission to referral and mortality. However, patients who were transferred had a significantly longer inpatient ward stay $(<0.001)$ but shorter ICU stay $(p<0.012)$.

\section{DISCUSSION}

To the best of our knowledge, this is the first report in published literature on the role of remote care (ie, huband-spoke model) approach for the management of SAP patients in the UK. This hub-and-spoke model facilitates the management of the majority of patients with SAP in secondary care setting. Only 29\% warranted transfer to tertiary care centre, predominantly for endoscopic management of PFCs. The inpatient stay was significantly longer for patients transferred to our service reflecting the severity of illness. An evidence-based approach with a low threshold for transfer to tertiary care centre has resulted in lower mortality compared with previously published studies. ${ }^{1-3}$

Studies have shown a benefit from centralisation of care in high-volume centres for patients with SAP as mortality and hospital stay decreased with an increased volume. ${ }^{1-13}$ However, the lack of a central point of contact to facilitate the care can be a significant hurdle in the management of this group of patients. Our weekly MDM setup has physical presence of pancreatologists, pancreatic surgeons, radiologists, a specialist dietician and pancreatitis specialist nurse. The unique feature of this service at the Freeman hospital was the specialist pancreatitis nurse who facilitated completion of referral data, including baseline characteristics and imaging, formally provides feedback to the referring team on the same day. This ensures a prompt, efficient and optimal management plan of the patients in regional hospitals.

Appropriate nutritional supplementation is a key intervention resulting in favourable outcomes in patients with SAP. One of the criticisms from the NCEPOD report concerned nutritional assessment, which was found to only have taken place in $67 \%$ of patients admitted with pancreatitis. ${ }^{1}$ Our dietetic team input ensures that the nutritional status is addressed and adjusted as required on a weekly basis and was addressed in $100 \%$ of our referred cases. Inappropriate nutrition was only observed in only $1 \%$ of the patients who were transferred.

The MDM is especially focused on the clinical condition of the patient, the severity of the pancreatitis and the type of drainage required depending on location and maturity of the collection. Endoscopic drainage is the modality of choice for drainage of walled-off necrosis. ${ }^{2}{ }^{3}$ The NCEPOD report showed that $30 \%$ underwent surgical treatment for acute pancreatitis complications which is higher than in our experience. Seventy-five per cent of the centres participating in the NCEPOD report reported to have access to a form of regional care network for pancreatitis but it is unclear as to whether those networks consisted of mainly surgical teams, which could explain the high rate of surgical treatments found in the report. ${ }^{1}$ Unpublished data between 2013 and 2015 from our unit showed a significant change with a reduction in surgical intervention from $13 \%$ to $7 \%$. This change in practice is in keeping with the published literature favouring minimal invasive approaches, which include endoscopic and percutaneous treatment. ${ }^{8-20}$ However, these decisions and optimal treatment plan are only possible after robust discussions in an MDT comprising of all relevant specialists.

Another important aspect of the care of these patients is the role for antibiotics. The NCEPOD report found a particularly worrying aspect of over $60 \%$ of acute pancreatitis patients prescribed antibiotics despite lack of evidence in preventing infectious complications. ${ }^{1-3}$ This was a major criticism of the care of pancreatitis in the UK with subsequent specific statement on antibiotic use in the recent NICE-guidelines for acute pancreatitis. ${ }^{2}$ In our study, $66 \%$ of patients referred were on antibiotics of whom only $31(56 \%)$ were identified to have had positive cultures at referral. The network had advised the referring centres to not prescribe antibiotics in the remaining $44 \%$ of patients.

This study has a few limitations. Despite the available data on the total of patients admitted locally with acute pancreatitis, the $6.1 \%$ referral rate could be an underestimate as the onus to refer was on the local team. However, we continue to educate the secondary care teams during our weekly MDT meetings and raise awareness of timely referral of patients with SAP to our unit for specialist input including the role of antibiotic therapy and adequate nutrition. In 
addition, obtaining complete data on patient who were not transferred can be challenging. There was also a delay in transfer to our centre by a median of 3 days; however, what effect this may have had on patient outcomes is unknown. In the future with the rapidly developing state of the art audio visual technology; we hope to make the MDT more accessible to the referring clinician who can link in live into the meeting and provide more detailed input about the patient rather than relying on the information on the referral form. This will enable us to provide a more robust and efficient management plan for this sick group of patients.

However, the strength of our study was the dedicated MDT including a dedicated specialist pancreatitis nurse which provides a standardised approach to each patient referred with all areas of pancreatitis care considered. This has not been previously reported and is unique to our service. We believe that the limitations did not affect the workflow and treatment of severe pancreatitis via our regional MDM. In addition, there was continuity of care due to the weekly discussions which triggered the need to transfer the patients to our unit for specialist input.

In conclusion, this is the first reported study to describe the role of a regional network for the management of SAP with relevant outcomes. This service is aided by a multispecialty core team providing regular support to a wide ranging population of patients with SAP. This unique hub-and-spoke model allows some patients with severe disease to remain at their base hospital but to benefit from the expertise at the specialist centre and this allows ICU beds at the tertiary centre to remain available, for complex, elective cases while ensuring that specialist management is still available for SAP patients. The added advantage is that patients remain close to home which is beneficial both for the patient and the family. Prospective assessment in other pancreatic units including economic analysis would be useful to determine if this model is transferable and beneficial.

\section{Author affiliations}

${ }^{1}$ HPB Medicine \& Translational and Clinical Research Institute, Newcastle University, Freeman Hospital, Newcastle upon Tyne, UK

${ }^{2}$ HPB Unit, Freeman Hospital, Newcastle upon Tyne, UK

${ }^{3}$ Newcastle Nutrition, Freeman Hospital, Newcastle upon Tyne, UK

${ }^{4}$ HPB Surgical Unit, Freeman Hospital, Newcastle upon Tyne, UK

${ }^{5}$ Department of Radiology, Freeman Hospital, Newcastle upon Tyne, UK

${ }^{6}$ Department of Intensive Care Medicine, Freeman Hospital, Newcastle upon Tyne, UK

Contributors MKN conceived the idea for the study, planning the work, collected and analysed data and wrote the manuscript. RMC, NLHB, JSL and KWO have been actively involved in the development of the study, management of patients, conduscting the study and help with the manuscript. DB, SY, JLL, GS, JJF, DC and SP have been actively involved in the management of the patients and planning the study.

Funding The authors have not declared a specific grant for this research from any funding agency in the public, commercial or not-for-profit sectors.
Competing interests None declared.

Patient consent for publication Not required.

Ethics approval Ethical approval from an institutional review body was not required for this study. Data collection was performed as part of on-going service evaluation of our service. Normal NHS clinical audit practice was observed. All aspects of the study were conducted in accordance with the Declaration of Helsinki 1964, as revised in Tokyo 2004.

Provenance and peer review Not commissioned; externally peer reviewed.

Data availability statement Data are available in a public, open access repository. All data relevant to the study are included in the article or uploaded as online supplemental information. All data have been included in the manuscript.

Supplemental material This content has been supplied by the author(s). It has not been vetted by BMJ Publishing Group Limited (BMJ) and may not have been peer-reviewed. Any opinions or recommendations discussed are solely those of the author(s) and are not endorsed by BMJ. BMJ disclaims all liability and responsibility arising from any reliance placed on the content. Where the content includes any translated material, BMJ does not warrant the accuracy and reliability of the translations (including but not limited to local regulations, clinical guidelines, terminology, drug names and drug dosages), and is not responsible for any error and/or omissions arising from translation and adaptation or otherwise.

Open access This is an open access article distributed in accordance with the Creative Commons Attribution Non Commercial (CC BY-NC 4.0) license, which permits others to distribute, remix, adapt, build upon this work non-commercially, and license their derivative works on different terms, provided the original work is properly cited, appropriate credit is given, any changes made indicated, and the use is non-commercial. See: http://creativecommons.org/licenses/by-nc/4.0/.

\section{ORCID iDs}

Manu K Nayar http://orcid.org/0000-0002-1196-3406

John S Leeds http://orcid.org/0000-0002-5140-6225

\section{REFERENCES}

1 O'Reilly DA, McPherson SJ, Sinclair MT, et al. Lessons from a national audit of acute pancreatitis: A summary of the NCEPOD report 'Treat the Cause'. Pancreatology 2017;17:329-33.

2 Pancreatitis NICE guideline, 2018. Available: https://www.nice.org. uk/guidance/ng104/resources/pancreatitis

3 Arvanitakis M, Dumonceau J-M, Albert J, et al. Endoscopic management of acute necrotizing pancreatitis: European Society of gastrointestinal endoscopy (ESGE) evidence-based multidisciplinary guidelines. Endoscopy 2018;50:524-46.

4 Johnson CD, Besselink MG, Carter R. Acute pancreatitis. BMJ 2014;349:g4859.

5 Singh VK, Bollen TL, Wu BU, et al. An assessment of the severity of interstitial pancreatitis. Clin Gastroenterol Hepatol 2011;9:1098-103.

6 Johnson CD, Abu-Hilal M. Persistent organ failure during the first week as a marker of fatal outcome in acute pancreatitis. Gut 2004;53:1340-4.

7 van Brunschot S, Hollemans RA, Bakker OJ, et al. Minimally invasive and endoscopic versus open necrosectomy for necrotising pancreatitis: a pooled analysis of individual data for 1980 patients. Gut 2018;67:697-706.

8 Bakker OJ, van Santvoort HC, van Brunschot S, et al. Endoscopic transgastric vs surgical necrosectomy for infected necrotizing pancreatitis: a randomized trial. JAMA 2012;307:1053-61.

9 Hollemans RA, Bakker OJ, Boermeester MA, et al. Superiority of step-up approach vs open necrosectomy in long-term followup of patients with necrotizing pancreatitis. Gastroenterology 2019;156:1016-26.

10 Banks PA, Bollen TL, Dervenis C. Classification of acute pancreatitis-2012: revision of the Atlanta classification and definitions by international consensus acute pancreatitis classification Working group. Gut 2013;62:102-11.

11 Wu BU. The impact of hospital volume on outcomes in acute pancreatitis: a case for centers of excellence? Gastroenterology 2009;137:1886-8.

12 Murata A, Matsuda S, Mayumi T, et al. Effect of hospital volume on clinical outcome in patients with acute pancreatitis, based on a national administrative database. Pancreas 2011;40:1018-23.

13 van Grinsven J, van Brunschot S, van Santvoort HC, et al. The value of a 24/7 online nationwide multidisciplinary expert panel for acute necrotizing pancreatitis. Gastroenterology 2017;152:685-8. 
14 van Brunschot S, van Grinsven J, van Santvoort HC, et al. Endoscopic or surgical step-up approach for infected necrotising pancreatitis: a multicentre randomised trial. Lancet 2018;391:51-8.

15 Charnley RM, Lochan R, Gray H, et al. Endoscopic necrosectomy as primary therapy in the management of infected pancreatic necrosis. Endoscopy 2006;38:925-8.

16 Huggett MT, Oppong KW, Pereira SP, et al. Endoscopic drainage of walled-off pancreatic necrosis using a novel self-expanding metal stent. Endoscopy 2015;47:929-32.

17 Bekkali NLH, Nayar MK, Leeds JS, et al. A comparison of outcomes between a lumen-apposing metal stent with electrocautery-enhanced delivery system and a bi-flanged metal stent for drainage of walledoff pancreatic necrosis. Endosc Int Open 2017;5:E1189-96.
18 Bang JY, Arnoletti JP, Holt BA, et al. An endoscopic transluminal approach, compared with minimally invasive surgery, reduces complications and costs for patients with necrotizing pancreatitis. Gastroenterology 2019;156:1027-40.

19 Garg PK, Zyromski NJ, Freeman ML. Infected necrotizing pancreatitis: evolving interventional strategies from minimally invasive surgery to endoscopic Therapy-Evidence mounts, but one size does not fit all. Gastroenterology 2019;156:867-71.

20 van Santvoort HC, Besselink MG, Bakker OJ, et al. A step-up approach or open necrosectomy for necrotizing pancreatitis. $N$ Engl J Med 2010;362:1491-502. 